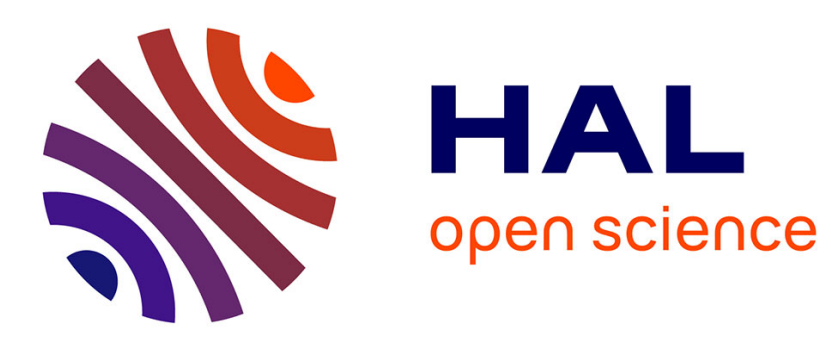

\title{
Histoire des bibliothèques françaises, tome IV, "Les bibliothèques au XXe siècle, 1914-1990", sous la direction de Martine Poulain
}

\author{
Jean-Marie Privat
}

\section{- To cite this version:}

Jean-Marie Privat. Histoire des bibliothèques françaises, tome IV, " Les bibliothèques au XXe siècle, 1914-1990 ", sous la direction de Martine Poulain. Pratiques: linguistique, littérature, didactique, 1993, 80, pp.116-125. 10.3406/prati.1993.2361 . hal-03180259

\section{HAL Id: hal-03180259 \\ https://hal.univ-lorraine.fr/hal-03180259}

Submitted on 24 Mar 2021

HAL is a multi-disciplinary open access archive for the deposit and dissemination of scientific research documents, whether they are published or not. The documents may come from teaching and research institutions in France or abroad, or from public or private research centers.
L'archive ouverte pluridisciplinaire HAL, est destinée au dépôt et à la diffusion de documents scientifiques de niveau recherche, publiés ou non, émanant des établissements d'enseignement et de recherche français ou étrangers, des laboratoires publics ou privés. 
Histoire des bibliothèques françaises, tome IV, « Les bibliothèques au XXe siècle, 1914-1990 », sous la direction de Martine Poulain

Jean-Marie Privat

\section{Citer ce document / Cite this document :}

Privat Jean-Marie. Histoire des bibliothèques françaises, tome IV, « Les bibliothèques au XXe siècle, 1914-1990 », sous la direction de Martine Poulain. In: Pratiques : linguistique, littérature, didactique, n80, 1993. pp. 116-125;

doi : https://doi.org/10.3406/prati.1993.2361

https://www.persee.fr/doc/prati_0338-2389_1993_num_80_1_2361

Fichier pdf généré le 13/07/2018 


\title{
NOTES DE LECTURE
}

\author{
Jean-Marie PRIVAT
}

Histoire des bibliothèques françaises, tome IV, « Les bibliothèques au $X X^{e}$ siècle, 1914-1990 », sous la direction de Martine Poulain,

Paris, Editions du Cercle de la Librairie-Promodis, 1992, 793

ABF, ADLP, BBF, BCP, BDF, BPI, CAFB, CARD, CSB, DLL, ENSB, JPL, etc.; Eugène Morel, Ernest Coyecque, Marguerite Durand, Claire Huchet, Madeleine Gruny, Mathilde Leriche, Paule Salvan, Paul Poindron, Henri Vendel, Julien Cain, Alice Garrigoux, Jean Hassenforder, H.-J. Martin, Jean Gattegno, Michel Melot, etc.; " liste Otto », " note 4 », « bibliothèque circulante », " modèle anglo-saxon », " normes bibliothéconomiques », " rapport Vandevoorde », " lecture publique », " catalogue collectif national », etc.; 1906 (création de l'Association des bibliothécaires français), 1931 (Congrès international de la lecture publique à Alger), 1938 (lancement du premier bibliobus), 1972 (année internationale du livre organisée par I'UNESCO), 1982 (Rapport PingaudBarreau sur le livre et la lecture), 1992 (nouveaux statuts des personnels des bibliothèques de la fonction publique territoriale), etc.

Autant de sigles familiers, de noms célèbres, d'expressions usuelles, de dates historiques, parmi des centaines d'autres, qui font et fondent la culture professionnelle des bibliothécaires d'aujourd'hui mais qui, sans doute, n'évoquent rien de bien précis chez la plupart des professeurs.

Or, ce beau livre de bibliothèque sur les bibliothèques (1) d'une très grande

(1) Le tome I est consacré aux Bibliothèques médiévales ( $\mathrm{VI}{ }^{\mathrm{e}}$ siècle-1530), le tome II aux Bibliothèques sous l'Ancien Régime (1530-1789) et le tome III aux Bibliothèques de la Révolution et du XIXe siècle (1789-1914). 
lisibilité (chronologie, index, nombreux encadrés (2)), d'une présentation extrêmement soignée et offrant de très nombreuses illustrations met en évidence avec force et force science ( 70 collaborateurs, nombreuses bibliographies sélectives) deux réalités fortes qui importent aux didacticiens. II manifeste d'une part combien livres, lecteurs et lectures sont modelés par l'histoire, d'autre part combien sont liées, bon gré mal gré, histoire de la lecture scolaire et histoire de la lecture publique, espace éducatif et espace culturel. L'ignorer reviendrait à accepter une définition restrictive et statique, aussi illusoire que chagrine, de notre travail d'enseignant.

Dans les pages qui suivent nous rendrons compte essentiellement de l'évolution des bibliothèques publiques et des mutations des bibliothèques pour les enfants ou pour la jeunesse. Nous laisserons aux lecteurs le loisir de découvrir par eux-mêmes les contributions qui décrivent par exemple le traitement technique des ressources documentaires ou celles qui nous font parcourir les chemins qui mènent de la bibliothèque à l'œuvre littéraire.

\section{Politiques du livre : de la léthargie à la fureur}

Contrairement aux représentations conventionnelles ou traditionnelles, les pratiques de lecture sont traversées de luttes, symboliques ou non, incessantes. Ces luttes qui déterminent fortement les conditions voire les enjeux de la pratique des livres sont multi-formes.

«La Bibliothèque d'Epinal sera fermée jusqu'à nouvel ordre » (L'Express de l'Est, 24 octobre 1940) : la violence physique et idéologique est la forme la plus voyante et la plus tragique d'une mise hors-jeu et des livres et des lecteurs. Dans une contribution fort émouvante et très documentée - Les bibliothèques dans la tourmente - Marie Kühlmann raconte «l'exode des livres » et «les nouveaux maîtres de la lecture », " les permissions de lire » et "la vengeance contre les livres ».

Hélas, la manipulation totalitaire du lecteur n'est pas nécessairement une parenthèse dans l'histoire ; que l'on songe aux imprécations intégristes contre la lecture des œuvres de Rushdie, aujourd'hui et partout, aux interdits pesant sur la possession de la Bible ou du Capital, hier et ailleurs.

L'apathie de l'Etat et des collectivités peut être une autre manière, délibérée ou non, de rendre difficile l'exercice de la lecture. Le "Constat des carences " (1914-1945) occupe ainsi toute la première partie de l'ouvrage.

En 1929 par exemple, 80\% des municipalités françaises allouaient moins de 1 franc par habitant et par an aux activités de leurs bibliothèques respectives, près de la moitié y consacraient moins de 50 centimes. Les achats de livres restaient donc voisins de zéro : 20 centimes par habitants à Lyon, 14 à Toulouse, 9 à Bordeaux, 5 à Marseille. Un inspecteur général résume amèrement la situation en ces termes:

(2) On lira par exemple les encadrés sur Georges Bataille, bibliothécaire, sur La bibliothèque Tourgueniev, sur Lucien Herr, administrateur de la bibliothèque de l'Ecole normale supérieure de 1888 à 1926, sur La Joie par les livres, sur L'image des bibliothèques dans la fiction au XX siècle, sur La Bibliothèque publique d'information (B.P.I.), sur La Médiathèque de la Cité des sciences et de l'industrie, sur La Bibliothèque de l'Institut Mémoires de l'édition contemporaine. 
"Beaucoup de municipales ne sont que des cimetières de livres, où les volumes de philosophie périmée, de théologie désuète, d'histoire vieillie, trompent les visiteurs par leurs belles reliures et donnent aux municipalités l'illusion qu'elles ont une bibliothèque ».

Autrement dit, les pouvoirs publics ne passèrent que très lentement " de l'indifférence à l'intérêt »(P. Ory).

Le manque d'empressement des autorités locales et centrales fut cependant vivement combattu par des organisations professionnelles (Association des Bibliothécaires Français, Association pour le Développement de la Lecture Publique), par des initiatives locales et par des personnalités hors du commun. Gérard Collon par exemple, directeur de la B.M. de Tours, estimait déjà en fin politique qu'un service qui marche bien et a une bonne réputation auprès du public fait honneur à l'administration régnante et, par ricochet, lui assure des budgets moins misérables. Aussi, décida-t-il, dés 1926, d'ouvrir la salle de lecture le dimanche et en une seule année le nombre d'ouvrages consultés doubla, ou peu s'en faut.

En dépit des restrictions budgétaires et de tous les conservatismes, l'entredeux-guerre a ainsi correspondu malgré tout à une période de débats intenses et d'expériences intéressantes où la cause des bibliothèques ouvertes à tous, selon le modèle anglo-saxon (accueil des publics, gratuité, élargissement du fonds, libre accès aux rayons, professionnalisation des personnels) a commencé à progresser. Mais ce n'est que bien plus tard que purent être dépassés les problèmes de simple fonctionnement (horaires d'ouverture inadéquats, personnel peu nombreux, règlements désuets, locaux inadaptés, collections lacunaires) et mieux pris en compte jusque dans les campagnes les besoins modernes d'ordre éducatif, récréatif, culturel et documentaire.

On lira avec un intérêt particulier les articles sur les premiers congrès internationaux d'où les « modernistes » revenaient pleins d'exemples décisifs et d'ardeur retrouvée pour adhérer à une « internationale » des amis du livre et les monographies de $\mathrm{N}$. Richter sur ces précurseurs admirables de persévérance et de sens du bien commun qui « ont fait la lecture publique ». Ces hommes (La Fontaine et Otlet - la CDU, La Classification Décimale Universelle, c'est eux Morel, Coyecque, Lemaître, Henriot) ont milité pour qu'on passe d'un encadrement de la lecture à une éducation du lecteur, d'une conception morale à une conception culturelle du livre, d'un service pour privilégiés à un service public.

On regrettera cependant qu'une contribution particulière ne se soit pas attachée à décrire plus systématiquement les inévitables tensions internes au camp des modernistes qui n'avaient, on s'en doute, ni les mêmes origines et les mêmes trajectoires culturelles, ni les mêmes expériences professionnelles, ni les mêmes responsabilités officielles. La difficulté objective et historique voire idéologique pour acclimater en France un modèle étranger («américain ») ne pouvait en effet que mettre à vif ces dissensus partiels ou potentiels, jusque et y compris dans le contexte des politiques culturelle et sociale du Front Populaire.

Dans les années trente, la lecture publique subit de violentes attaques des professionnels de la lecture savante et dut faire face à « la résistance pratique développée au quotidien " par de nombreux bibliothécaires aussi obscurs que prisonniers de la routine. Certes, contre les « conservateurs ", les « modernes " présentaient un front cohérent, mais pas vraiment homogène. On savait gré à la verve d'Eugène Morel d'avoir dénoncé une situation n'ayant que trop duré, 
mais l'on s'empressa de lui substituer un ton plus mesuré : «Ces écrits font scandale, y compris dans les milieux novateurs ». Et la contradiction peut travailler les prises de positions d'une même personnalité.

Au cœur de la fascination d'Ernest Coyecque pour les bibliothèques américaines, par exemple,

« il y a, prépondérante, la volonté de promouvoir une lecture de type instrumental, dont les périodiques seraient le support privilégié, autour d'une fonction primordiale : renseigner. Or une telle lecture paraît bien éloignée des habitudes françaises qui manifestent un goût marqué pour la fiction. Coyecque doit donc gérer la tension potentielle entre ces deux fonctions ainsi qu'une contradiction puisqu'il souhaite valoriser une fonction sous-représentée tout en prétendant que la bibliothèque doit, à la manière d'un commerce, répondre à la demande du public » (L. Léveillé, Fascinations étrangères et naissance de la "lecture publique ", pp. 174-177).

L'hommage historique dû aux pionniers de la lecture moderne ne gagne pas plus à laisser entendre que ces messieurs étaient "progressistes " en tout et pour tout. Même s'il faut resituer les positions et prises de positions dans le contexte social et professionnel de l'époque, on ne peut pas écrire sans autre forme de procès qu'Eugène Morel " sera le premier à recommander l'emploi de personnel féminin dans les bibliothèques » (N. Richter (3), p. 161).

En effet, l'auteur inspiré de Bibliothèques. Essai sur le développement des bibliothèques publiques et de la librairie dans les deux mondes (1909), l'auteur inoubliable de cette formule-manifeste "II nous faut des bibliothèques libres. Libres d'accès, libres d'esprit » s'est aussi interrogé sur «les femmes et la carrière des bibliothèques " :

"La femme peut-elle être dans les bibliothèques l'égale de l'homme, peut-elle administrer, diriger, fonder ? A travail égal doit-elle avoir salaire égal ? ».

A la question touchant à l'exercice de hautes fonctions au sein de la bibliothèque, il est répondu que le « souci de l'intérêt général, le travail absorbant et désintéressé " exigés par ce type de responsabilité est peu compatible le plus souvent avec les " charges maternelles et familiales ", avec l'exercice de

" ce métier de femme auquel nous devons la conservation du genre humain [...]. Qu'est-ce que le sexe féminin aura gagné à ce que les quelques douzaines de places honorées soient femelles au lieu d'être mâles ? Rien que de la vanité ».

A la question relative au salaire, Morel explique que " sans déroger à l'orthodoxie syndicale, on peut admettre quelques heures de travail mal payées pour une femme à laquelle ses obligations de ménage interdisent tout travail régulier ". On peut obtenir des femmes un " service régulier, docile et minutieux » ; certaines tâches sont d'ailleurs particulièrement appropriées à leurs "facultés » et toute une "série de pauvres diables auxquels la bibliothèque fournit un salaire d'appoint » (instituteurs, comptables, retraités) seraient avantageusement remplacés dans "leur besogne essentiellement féminine »

(3) Cette réserve sur un point particulier d'histoire sociale des bibliothèques ne doit pas faire oublier l'immense, le précieux et le passionnant travail de Noë RICHTER sur les conditions historiques des pratiques de lecture; on lira avec intérêt et profit Les Bibliothèques populaires (Paris, Cercle de la Librairie, 1978), L'Education ouvrière et le livre, Le Mans, Université du Maine, 1982) et, synthèse des précédents, les deux volumes consacrés à La Lecture et ses institutions (t. 1, La lecture populaire, 1700-1918, Editions Plein Chant / Université du Maine, 1987 ; t. 2, La lecture publique, 1919-1989, Editions Plein Chant, 1989. 
par des demoiselles et des dames : distribution des livres, copies de catalogues, " et surtout là où les emprunteurs sont des enfants » (E. Morel, La Librairie publique, Paris, Colin, 1910, pp. 294-297).

Reste qu'avec ces pionniers, bien sûr, s'est affirmé " un discours plus technique sur les bibliothèques, privilégiant les structures et le fonctionnement de l'institution, longtemps négligé au profit d'un discours vague et moralisateur sur le « bon » livre. Ainsi, lentement, la bibliothèque s'est dégagée de l'inspiration purement philanthropique et morale pour bénéficier de nouvelles considérations telles que le droit à la lecture ou le devoir incombant à l'Etat de promouvoir un service public de lecture » (Léveillé, p. 177).

La deuxième grande partie de l'ouvrage nous fait découvrir la "Naissance des politiques publiques, 1945-1975 ". II s'agit de choisir, au sortir de la guerre, entre le libéralisme ou le dirigisme culturels.

Dans la conception libérale «pure », la culture, contrairement à l'instruction, est réputée relever du seul domaine privé. A ce titre, elle ne saurait faire l'objet d'autre sollicitude que celle qui assure à chacun d'exercer librement, et si possible dans les meilleures conditions, son droit à la culture en général, son « right to read » en particulier.

Une autre politique, dans un pays très marqué par la lutte séculaire de l'Eglise et de l'Ecole, consisterait au contraire à postuler que les conduites culturelles sont à gagner contre l'ignorance et contre l'obscurantisme. Aussi, on ne s'étonnera pas si l'ordonnance du 2 novembre 1945, dans un souci d'unanimisme et d'égalitarisme culturels (dont J. Guéhenno fut un temps le héros), se proposait de " mettre à la disposition de tous les travailleurs, même isolés, un fonds de livres d'études » (D. Lindenberg, «Les bibliothèques dans les politiques éducatives et culturelles », pp. 253-271).

La grande réforme liée à cette période et aux espoirs de la Libération sera en effet la création des bibliothèques centrales de prêt. Les BCP vont symboliser " une politique de démocratisation du savoir » qui puise ses sources dans Michelet et Hugo ; de même que les républicains du $\mathrm{XIX}^{\mathrm{e}}$ siècle avaient voulu faire pénétrer l'instruction dans le moindre village, leurs successeurs de 1945 se montraient soucieux de faire reculer l'inégalité devant le livre entre villes et campagne, Paris et province. Mais en fait, l'Etat fut longtemps défaillant et en 1967 seuls une quarantaine de départements étaient desservis.

De leurs côtés, les B.M. vont connaître pendant de longues années encore une dégradation certaine : "Tout est à faire 》 déclare en 1966 G. Pompidou, Premier Ministre. Et au milieu des années soixante dix, même si une Direction des Bibliothèques existe depuis plus de vingt ans, la France n'est « toujours pas dotée d'un réseau de bibliothèques capable de répondre aux attentes et aux besoins d'une société qui change plus vite qu'elles » (M. Poulain, "Introduction ", p. 5). Les BCP ne touchent que $10 \%$ de la population visée et les BM ne desservent que $4,6 \%$ de la population urbaine. Ne parlons pas du sort des bibliothèques universitaires.

Les mouvements associatifs et militants, là encore, travaillent avec énergie pour que les bibliothèques soient un peu plus à la hauteur de leur tâche éducative et culturelle. Les militants de la CGT, Travail et Culture, luttent pour des lectures instructives ou sainement distractives ; Begnino Cacérès, secrétaire général de Peuple et Culture prônent l'animation autour du livre, dans l'entreprise : « exposi- 
tions, causeries, excursions, voyages, ventes de livres, invitations à participer à la gestion de la bibliothèque, listes d'ouvrages thématiques doivent être autant de médiations propres à enrichir les connaissances ouvrières et à aider à la constitution de sens dans les lectures » (M. Poulain, « Livres et lecteurs », p. 276). C'est ce modèle d'animation culturelle qui se répandra plus tard dans les B.M.

En dépit de divergences idéologiques profondes, laïcs convaincus (Ligue de l'enseignement) ou croyants sincères (Culture et Bibliothèques pour tous) les militants du livre, professionnels ou bénévoles, sont tous profondément humanistes. Le livre aurait la vertu incomparable d'offrir un plaisir de qualité et / ou d'être un ferment d'émancipation sociale et intellectuelle. On comprend que ces valeurs se forgent dans la lutte contre "la littérature facile » d'une part, contre les progrès de la culture de masse d'autre part. Aussi, le public privilégié des bibliothèques associatives est-il celui de lecteurs qui prisent une culture moyenne (à sa portée), à égale distance des lectures lettrées et des lectures populaires.

La volonté de faire partager les « joies culturelles » (B. Cacérès) au plus grand nombre rendent indispensable la connaissance du public et donc le développement des sciences sociales. Ce sont alors les premières recherche-actions, "pour le progrès culturel de la société ", de Joffre Dumazedier et Jean Hassenforder et le Que Sais-Je de R. Escarpit sur La Sociologie de la littérature (1958).

De cette même période (1960) date la première enquête un peu importante sur la lecture et le livre en France (commandée par le Syndicat National des Editeurs et non par l'Education nationale) et un débat passionné sur le livre de poche (1964-1965) :

«Les uns craignent une dévalorisation du rapport à l'écrit par une banalisation trop forte du support du texte. Les autres espèrent au contraire de cette banalisation un plus grand partage culturel » (M. Poulain, p.285).

On sait que les uns et les autres se trompaient puisque le livre reste un bien culturel légitime et que l'accessibilité du " poche " a surtout profité aux lecteurs déjà confirmés.

Les quinze dernières années - "La place nouvelle des bibliothèques, 19751990 » sont celles de l'envol des bibliothèques qui deviennent un formidable enjeu politique, culturel et social (songeons à la découverte de l'illettrisme et aux discours surdramatisés qui l'accompagnent). Si les Maisons de la Culture voulues par $\mathrm{A}$. Malraux ne prévoyaient pas de bibliothèques dans leurs murs, la dernière période voit s'intensifier de façon spectaculaire, sous l'impulsion de l'Etat et des collectivités territoriales (loi de décentralisation aidant), les politiques de développement des médiathèques et des Maisons du Livre. En moins de vingt ans, le nombre de BM et le nombre de livres prêtés doublent, les crédits d'acquisition, la surface des locaux ou encore le nombre d'inscrits en valeur absolue triplent. « La » bibliothèque ou plutôt « les » bibliothèques vivent désormais avec leur temps.

Alors que dans la période précédente la « méfiance » à l'égard du roman, lecture de pure évasion et en tout cas moins recommandable que l'instructif documentaire, est une attitude " très prégnante à l'époque chez les bibliothécaires » (M. Poulain, p. 289), désormais, les bibliothèques sont résolument multi-média et multi-public. L'urbanisation de la France, l'accroissement de la scolarisation, la montée en puissance des industries culturelles, l'explosion des technologies nouvelles, l'évolution même des conceptions éducatives (accès aux documents, travail autonome) et culturelles (pluralité des références, forma- 
tion continue) expliquent sans doute largement le brutal développement des bibliothèques publiques.

Ainsi, " encyclopédisme et syncrétisme, modernité et confort du lieu, liberté des usages sont les nouvelles valeurs promues par une offre qui cherche à faire la preuve que le divertissement peut être culturel, qui ne veut plus guider mais accompagner, qui accepte de former mais veut surtout informer, et proposer des libres parcours où chacun puisera selon ses inclinations et motivations " (M. Poulain, pp.6-7).

Cette volonté de démocratisation des conditions de l'accès aux livres est obnubilée par l'augmentation de la masse des consommateurs de culture ; contrairement aux périodes précédentes où la qualité des lectures était un sujet de préoccupation, c'est désormais le nombre de lecteurs qui obsèdent les décideurs politiques et les prescripteurs du livre. II faut lire, furieusement... On compte beaucoup, mais on connaît toujours aussi peu les publics réels et les usages qu'ils font véritablement de l'offre de lecture publique, leurs profils et leurs profits (excepté à la B.P.I du Centre G. Pompidou qui s'est dotée d'un observatoire des publics) (4). Et pourtant les innombrables sondages sur les pratiques de lecture rappellent l'inégal partage du lire et l'enquête de 1989 sur les Pratiques culturelles des français montre que le nombre global de lecteurs de livres subit une inquiétante stabilisation (même si les catégories traditionnellement peu ou non liseuses lisent un peu plus) et que l'intensité générale des lectures décroît. Des sociologues parlent alors de la démocratisation culturelle comme de «la fin d'un mythe » (5). Le désappointement des ministères concernés et des médiateurs de la chose imprimée est donc grand car la fréquentation des bibliothèques ne paraît pas à la hauteur des investissements financiers et du volontarisme des politiques culturelles : malgré un doublement des pourcentages en quinze ans, $15 \%$ seulement de la population (adulte) desservie est inscrite aujourd'hui dans une bibliothèque municipale.

Mais en un sens, c'est bien parce que les bibliothèques sont assez soudainement entrées dans notre modernité culturelle de proximité et sont désormais considérées comme un authentique service public que se posent avec acuité leur appropriation par le plus grand nombre et donc, au fond, l'éducation culturelle du jeune lecteur.

\section{«L'usage des bibliothèques fait partie de l'éducation » (E. Morel)}

Les bibliothèques destinées aux enfants n'apparaissent en France qu'après la Grande Guerre. On sait que la lecture des jeunes était officiellement intégrée (depuis 1860) au monde de l'Ecole, tant bien que mal. Faute de crédit(s) public(s), à tous les sens du terme, les armoires-bibliothèques scolaires, malgré les efforts de nombreux instituteurs, voient leurs collections se périmer et leurs fonds se dépareiller bien vite. L'aile avant-gardiste des bibliothécaires prône alors la création de sections juvéniles dans les bibliothèques et la formation de bibliothécaires spécialisés. II faudra attendre 1919 et l'aide du Comité américain pour les régions dévastées de la France (CARD) pour que s'ouvre à Vic-sur-

(4) Les publications du Service des Etudes et de la recherche de la Bibliothèque Publique d'Information sont d'un très grand intérêt pour les enseignants qui veulent penser les lieux du livre en termes d'imposition de modèles de pratiques ou en termes de réceptions différenciées et d'appropriation personnelles d'espaces culturels. La liste bibliographique des ouvrages parus et le Bulletin d'Information sont à demander au Service des Etudes et de la recherche, BPI, 19, rue Beaubourg, 75197, Paris cedex O4.

(5) O. DONNAT, « Démocratisation culturelle : la fin d'un mythe », Esprit, 3-4, mars-avril 1991, pp. 65-82. 
Aisne, dans un département particulièrement meurtri par la guerre, la première salle de lecture pour enfants.

Peu à peu, quelques bibliothèques municipales commencent à reconnaître l'existence d'un public spécifique de jeunes lecteurs et l'entre-deux-guerre voit se développer les sections de lecture enfantine. Souvent ces espaces réservés aux enfants sont crées sur le modèle de L'Heure Joyeuse, à Paris, avec l'aide du Book Committee on Children's Libraries qui souhaite promouvoir des bibliothèques entièrement consacrées aux enfants. Ouverte en 1924 dans un secteur populaire du Quartier Latin la bibliothèque de L'Heure Joyeuse fut une référence, elle qui sur le plan local noua des relations toujours plus intenses avec les enseignants des écoles voisines (6).

Nombreux sont les éditeurs, les auteurs, les illustrateurs qui fréquentèrent cette bibliothèque inédite. Paul Faucher y testa en 1930 ses premiers albums du Père Castor. Pour les professionnels, des cours sur les bibliothèques enfantines et les livres d'enfants furent même donnés à Paris dès 1923. II est vrai que chez les plus progressistes le livre et la bibliothèque étaient considérés comme un enjeu de civilisation et de paix.

Ce type de bibliothèque fonctionnait sur le modèle américain - larges plages d'ouvertures, mobilier adapté aux usagers, fréquentation gratuite, mixité, livres en libre accès, choix d'œuvres modernes et tout public, heure du conte et autres animations culturelles. Ces lieux où les enfants devaient se sentir " chez eux " connurent d'emblée un réel succès, bien que les petits usagers fussent quelque peu désorientés dans les premiers temps par tant de libertés et de nécessaires initiatives culturelles.

Mais sur le plan national, par manque de moyens financiers et faute d'un véritable soutien politique, l'initiative pour créer des bibliothèques pour enfants s'essouffla assez vite. D'autant que du côté de l'Education nationale, on ne voyait pas toujours d'un très bon œil cette concurrence qui venait ébrécher une forme de monopole sur le livre. Une bonne illustration de cette relative méfiance est le débat sur le bibliobus " à l'américaine » qui, en privilégiant le contact direct avec le lecteur contourne l'école, et le bibliobus « à l'anglaise » qui utilise le système des caisses de livres laissées en dépôt aux bons soins des instituteurs. L'organisation de la desserte circulante des livres va se faire en utilisant la structure d'accueil, commode et économique, de l'école, source renouvelée de toute instruction. Les « modernistes » supportent mal cette « tutelle » scolaire qui leur semble peu propice à l'avènement de la lecture publique :

«D'une part la tutelle de l'école perpétuait une lecture primarisée, réservée aux élèves, anciens élèves et parents d'élèves [...]. D'autre part, la bibliothèque, présentée comme le prolongement de l'école dans une œuvre organique d'éducation, n'était jamais considérée comme un fin en soi, mais seulement comme un moyen d'encadrement post-scolaire » (Laure Léveillé, p. 174).

Au Congrès d'Alger (1931), E. Coyecque considère ainsi qu'à tout prendre il vaut mieux que la bibliothèque scolaire s'en tiennent à sa vocation pédagogique... On connaît la postérité de ce partage des responsabilités éducatives.

(6) Malgré les nombreux articles ou études consacrés à L'Heure Joyeuse, il est difficile de se faire une idée précise de la fréquentation réelle, en termes d'âge, de catégories sociales et de niveau dans le cursus scolaire, de ce type de bibliothèque. Cette mythologisation des origines a quelque chose d'agaçant.

Sur le dispositif didactique de L'Heure Joyeuse, voir dans ce même numéro la présentation qu'en font D. LELIĖVRE et M.-C. VINSON en annexe de leur article. 
Certes, les associations professionnelles iront de l'avant. L'Association pour le développement de la lecture publique (ADLP) par exemple est fondée en 1936 dans le but de rassembler bibliothécaires, écrivains, éditeurs, enseignants désireux de défendre la cause des bibliothèques et de la lecture publique. Mais il faudra attendre l'après-guerre pour que « les bibliothèques pour enfants aient les moyens institutionnels et matériels de se développer, généralement au sein des bibliothèques municipales » (V. Ezraty, Les premières heures des bibliothèques pour enfants, pp. 204-219).

$\mathrm{Au}$ milieu des années soixante, la place des enfants dans les B.M. est paradoxalement mineure. En effet, même si les discours de la lecture proclament l'importance de la lecture des jeunes et même si dès 1957 se multiplient dans les congrès internationaux des bibliothécaires les communications sur les "bibliothèques enfantines ", la pratique est, dans les faits, bien mal encouragée par l'offre et ses règlements :

\begin{abstract}
"L'accès aux sections de jeunes est toujours soumis à une réglementation [...]. Dans la majorité des cas, les enfants qui désirent fréquenter la bibliothèque doivent présenter une autorisation écrite ou être présentés par leurs parents pour lire sur place et emprunter des livres. II leur faut dans certains cas signer un engagement de respect du règlement. Leur carte d'inscription est obligatoire dans quelques sections, même pour venir emprunter sur place. Parfois, à la suite de demandes de parents qui tiennent à vérifier si leurs enfants sont bien venus à la bibliothèque, les cartes sont timbrées à la date de chaque venue du lecteur. Elle ne leur est rendue qu'à la sortie de la salle afin d'éviter un simple passage pouvant servir d'alibi » (7).
\end{abstract}

Cependant, la création en 1951 d'une option " bibliothèques pour enfants " ( « jeunesse et lecture publique » à partir de 1960) au CAFB (Certificat d'Aptitude aux Fonctions de Bibliothécaire), la naissance en 1963 de l'Association La Joie par les Livres (héritière en partie de l'expérience de L'Heure Joyeuse), l'intensification des stratégies éducatives et péri-scolaires des classes moyennes, la relance du débat sur les conditions d'exercice d'une certaine forme de démocratie culturelle (mai 1968), le redressement et l'enrichissement relatif du pays aussi, expliquent qu'on voit progressivement

\begin{abstract}
" des sections pour enfants plus vastes remplacer les petites salles aménagées auparavant dans les bibliothèques reconstruites après la guerre. Des équipements plus grands remplacent les petites bibliothèques pour enfants installées dans les quartiers et les ZUP afin de combler les lacunes les plus graves. Puis, à partir de 1970 , il paraît préférable de ne pas isoler les bibliothèques pour enfants, afin d'éviter une coupure des pratiques de lecture au moment du "passage " à la bibliothèque des adultes. En fait, les bibliothèques pour enfants s'avèrent, autour de 1975, des secteurs assez bien dotés en collections [...] et actifs. Le taux de pénétration des bibliothèques est deux fois plus fort chez les enfants que chez les adultes. » (H. Richard, Les bibliothèques municipales, p. 352).
\end{abstract}

La dernière période présente quatre caractéristiques majeures et nouvelles.

- Nombre de prescripteurs de lecture (parents, bibliothécaires, instituteurs) et d'éditeurs pour la jeunesse souhaitent aider au développement de l'autonomie relative de l'enfant-lecteur. Les espaces de lecture qui sont dès lors proposés

(7) M. BOUYSSI, «Les sections pour la jeunesse dans les bibliothèques municipales de province ", Bulletin des Bibliothèques de France, juillet, 1964, № 7, cité par M. POULAIN, op. cit., pp. 290-291. 
sont conçus, aménagés et animés pour que s'exercent cette liberté et cette responsabilité nouvelles.

- Les bibliothèques prennent acte de la multiplication des manières de lire et des supports de lecture ; elles deviennent des médiathèques incluant des sections jeunesse. En effet, la concurrence de la culture audio-visuelle a fait apparaître comme terriblement secondaires «les débats antérieurs sur livres à prescrire et livres à proscrire, entre lecture du livre ou du journal, lecture de formation ou d'information, lecture de travail ou lecture de loisir » (A.M. Chartier, De nouvelles définitions du lire, p. 520).

- Le goût de lire et le plaisir de lire sont les valeurs exhibées par les médiateurs du livre. L'offre de biens culturels et de services symboliques doit provoquer le bien-être culturel du lecteur, par opposition sans doute à l'ascétisme triste et contraint des apprentissages trop scolaires. Désormais, la quête du salut culturel exige que l'on abandonne « la morale du devoir qui portait à la suspicion généralisée contre l'agrément et l'agréable ». C'est au contraire "le devoir de plaisir » que l'éthique libérale ou permissive revendique. La conception puritaine de l'enfant, être de pulsions aussi destructrices que puissantes, est abandonnée et avec elle la conception de l'éducation comme une technique de dressage ou de forçage. L'enfant est crédité d'une nature bonne et l'acte éducatif « culturel » consistera à lui offrir des situations où il découvrira par exploration personnelle et progressive socialisation des plaisirs légitimes. Les frontières entre jeu et travail, devoir et plaisir seront brouillées et les apprentissages devront apparaître comme des plaisirs nécessaires, "subjectivement agréables et objectivement indispensables » comme dit Bourdieu (8). On pourra craindre cependant que ce libéralisme de l'offre, cette humeur anti-institutionnelle qui se soucie d'échapper à tout ce qui rappelle les hiérarchies et les classements, et par dessus tout les hiérarchies et les classements scolaires, ne s'inquiète pas suffisamment des conditions d'accès à un lieu et des conditions d'appropriation de ces valeurs. On regrette d'ailleurs de ne pouvoir lire une contribution qui se serait attachée spécialement à décrire et à problématiser les innombrables (et peut-être répétitives) expériences de didactique de l'offre culturelle dans les bibliothèques publiques.

- Enfin, on peut convenir que " c'est la fin du partage simple » (A.M. Chartier) qui dans la division du travail culturel attribuait aux bibliothécaires le public lisant et à l'école les apprenants ; la bibliothèque s'adjoint en effet les bébés- lecteurs des crêches et les pré-lecteurs des maternelles mais aussi s'intéresse aux lecteurs fragiles ou aux non-lecteurs. Toutefois, les bibliothécaires sont persuadés que pour mener à bien cette «pédagogie sociale » et culturelle de la lecture (A.-M. Chartier), la médiation de la seule bibliothèque ne suffit pas.

Dans le cadre d'un futur numéro de Pratiques sur « L'appropriation culturelle ", nous reviendrons sur les différences et les différends entre Ecoles et Bibliothèques et retracerons l'évolution historique et didactique des BCD et des CDI, eux aussi « lieux de mémoire » relevant d'une mémoire collective et d'une histoire professionnelle.

(8) P. BOURDIEU, La Distinction, critique sociale du jugement, Paris, éd. de Minuit, " Du devoir au devoir de plaisir ", pp. 422-431. 